\title{
Immunotherapy Is Associated with a Survival Benefit in Patients Receiving Chemotherapy for Metastatic Pancreatic Cancer
}

\author{
Jonathan J. Hue, Katherine Bingmer, ${ }^{1}$ Kavin Sugumar, ${ }^{1}$ Sarah C. Markt, ${ }^{2}$ Luke D. Rothermel, ${ }^{1}$
} Jeffrey M. Hardacre, ${ }^{1}$ John B. Ammori, ${ }^{1}$ Jordan M. Winter, ${ }^{1}$ and Lee M. Ocuin ${ }^{1, *}$

\begin{abstract}
Background: Immunotherapy (IT) has led to improved survival in several common cancers but success in pancreatic ductal adenocarcinoma (PDAC) has been limited. We analyzed if combination IT-chemotherapy (IT-CT) is associated with improved survival compared with chemotherapy alone (CT) in patients with metastatic PDAC. Methods: The National Cancer Database (2004-2016) was queried for patients who were diagnosed with metastatic PDAC. Patients were categorized by treatment group: CT only and IT-CT. Patients were excluded if they received radiation or a surgical procedure. The primary outcome was overall survival.

Results: A total of 59,289 patients were identified, of whom 58,947 (99.4\%) received CT and 342 (0.6\%) received IT-CT. The IT-CT group was younger, had fewer comorbidities, and was more often treated at an academic center. The utilization of multiagent $C T$ was similar between the groups. Median survival of patients treated with IT-CT was longer than CT alone (7.9 months vs. 6.3 months, $p=0.005)$. On multivariable analysis, receipt of IT-CT was associated with a survival advantage as compared with $C T$ (hazard ratio $=0.86,95 \%$ confidence intervals 0.76 0.97) when adjusting for demographics and type of $C T$ regimen.

Conclusion: In patients with metastatic PDAC, it appears that combination IT-CT may perhaps be associated with a survival advantage compared with $\mathrm{CT}$ alone.
\end{abstract}

Keywords: pancreatic cancer; immunotherapy; chemotherapy; stage IV disease

\section{Introduction}

Multiagent chemotherapy (CT) remains the backbone of systemic treatment for patients with pancreatic ductal adenocarcinoma (PDAC). ${ }^{1}$ FOLFIRINOX (5fluorouracil, leucovorin, irinotecan, oxaliplatin), gemcitabine with nab-paclitaxel, and gemcitabine with capecitabine have demonstrated a survival benefit over gemcitabine monotherapy in patients with metastatic PDAC. ${ }^{2-4}$ Despite modest advances in the effectiveness of chemotherapeutics, further combinations of existing chemotherapies are unlikely due to compounding side effect profiles. Multiagent CT regimens are associated with severe toxicities, which have been reported in the majority of patients receiving FOLFIRINOX. ${ }^{2,5}$ Alternative treatment strategies are needed. There has been improvement in overall survival of other solid tumor malignancies over the past decade, such as melanoma, renal cell carcinoma, lung cancer, and bladder cancer, with the introduction of therapy targeting various immune system checkpoints. ${ }^{6-9}$ Immunotherapy (IT) aims to boost a patient's immune system to enhance tumor recognition and destruction. ${ }^{10-14}$ Therapies

${ }^{1}$ Division of Surgical Oncology, Department of Surgery, University Hospitals Cleveland Medical Center, Cleveland, Ohio, USA.

${ }^{2}$ Department of Population and Quantitative Health Sciences, Case Western Reserve University School of Medicine, Cleveland, Ohio, USA.

*Address correspondence to: Lee M. Ocuin, MD, Division of Surgical Oncology, Department of Surgery, University Hospitals Cleveland Medical Center, 11100 Euclid Avenue, Cleveland, $\mathrm{OH}$ 44106, USA, E-mail: lee.ocuin@UHhospitals.org

(C) Jonathan J. Hue et al., 2021; Published by Mary Ann Liebert, Inc. This Open Access article is distributed under the terms of the Creative Commons License [CC-BY] (http://creativecommons.org/licenses/by/4.0), which permits unrestricted use, distribution, and reproduction in any medium, provided the original work is properly cited. 
aimed at modulating the immune response to cancer (i.e., cancer vaccines, oncolytic viruses, etc.) have been under investigation for decades, ${ }^{15-18}$ but current immune checkpoint inhibitors were first approved in 2011 after demonstrating a survival benefit among patients with metastatic melanoma. ${ }^{19}$ Unfortunately, no such success has been realized in the treatment of PDAC to date. ${ }^{20-26}$

A previous retrospective analysis of patients with PDAC who underwent a pancreatectomy in the National Cancer Database (NCDB) demonstrated an association between improved overall survival and adjuvant IT-CT as compared with adjuvant CT alone. ${ }^{27}$ To our knowledge, there has not been a report on the impact of IT in metastatic PDAC patients using a large administrative database. The primary aim of this study was to use the NCDB to determine if the addition of IT to CT was associated with a survival advantage in patients with metastatic PDAC. In addition, we aimed to identify potential disparities in receipt of IT based on sociodemographic characteristics.

\section{Materials and Methods}

Institutional assurances

This project was exempt from approval by the Institutional Review Board at our institution under Federal Regulation 45 CFR 46.101 (b).

\section{Data source}

The NCDB is a nationwide database, jointly maintained by the American College of Surgeons and the American Cancer Society. This database receives patient information from Commission on Cancer-accredited hospitals across the United States and contains deidentified information on $\sim 70 \%$ of all new malignancies diagnosed in the United States each year. The accuracy of the data reported, statistical analyses performed, and conclusions drawn are not monitored nor verified by the American College of Surgeons or American Cancer Society. The NCDB Participant User File data dictionary contains definitions of variables used in this study. ${ }^{28}$

\section{Patient cohort}

Adult patients diagnosed with PDAC were identified between 2004 and 2016, using International Classification for Diseases in Oncology (third edition) codes 8140 and 8500 . Only patients with metastatic (stage IV) disease at diagnosis were included. Patients were excluded from the analysis if they underwent a surgical procedure, if they received radiotherapy, if the type of CT ad- ministered (single-agent or multiagent) was not recorded, or if data on IT administration was missing (consort diagram, Fig. 1). For this analysis, we utilized data on sociodemographic (age, sex, race, insurance status, education quartile, income quartile, CharlsonDeyo comorbidity index) and clinical characteristics, facility type (academic, community, comprehensive community, integrated), and treatment information.

\section{Exposure}

Patients with PDAC were categorized into two treatment groups: those who received single- or multiagent CT alone or IT and CT (IT-CT). Within the NCDB, IT is defined as biological or chemical agents that alter the immune system or change the host's response to tumor cells. ${ }^{28}$

\section{Outcomes}

The primary outcome in this study was overall survival, defined as the number of months from the patient's date of diagnosis to either their date of death or last follow-up. Cancer-specific outcomes were not evaluated because the NCDB does not capture data pertaining to progression or cause of death.

\section{Statistical analyses}

Clinical and demographic data were compared between the treatment groups. The Wilcoxon ranksum test was used to compare continuous dependent variables. Pearson's chi-squared test was used to compare categorical variables. Multivariable logistic regression was used to determine the association between IT usage and demographic characteristics and clinical variables. Median survival was analyzed using the Kaplan-Meier estimate and compared by the log-rank test. Multivariable Cox proportional hazards regression models were used to calculate hazard ratios (HRs) and 95\% confidence intervals (95\% CI) for the association between the treatment groups and survival, adjusting for sex, age, race (white, other), type of hospital (academic, other), insurance status (private, other), and Charlson-Deyo comorbidity score $(0, \geq 1)$. The NCDB does not include survival data for patients diagnosed in 2016, thus these patients were not included in survival analyses. StataSE v16.0 (Statacorp LLC, College Station, TX) was used for statistical analyses. A $p$-value $<0.05$ was used to indicate statistical significance. 


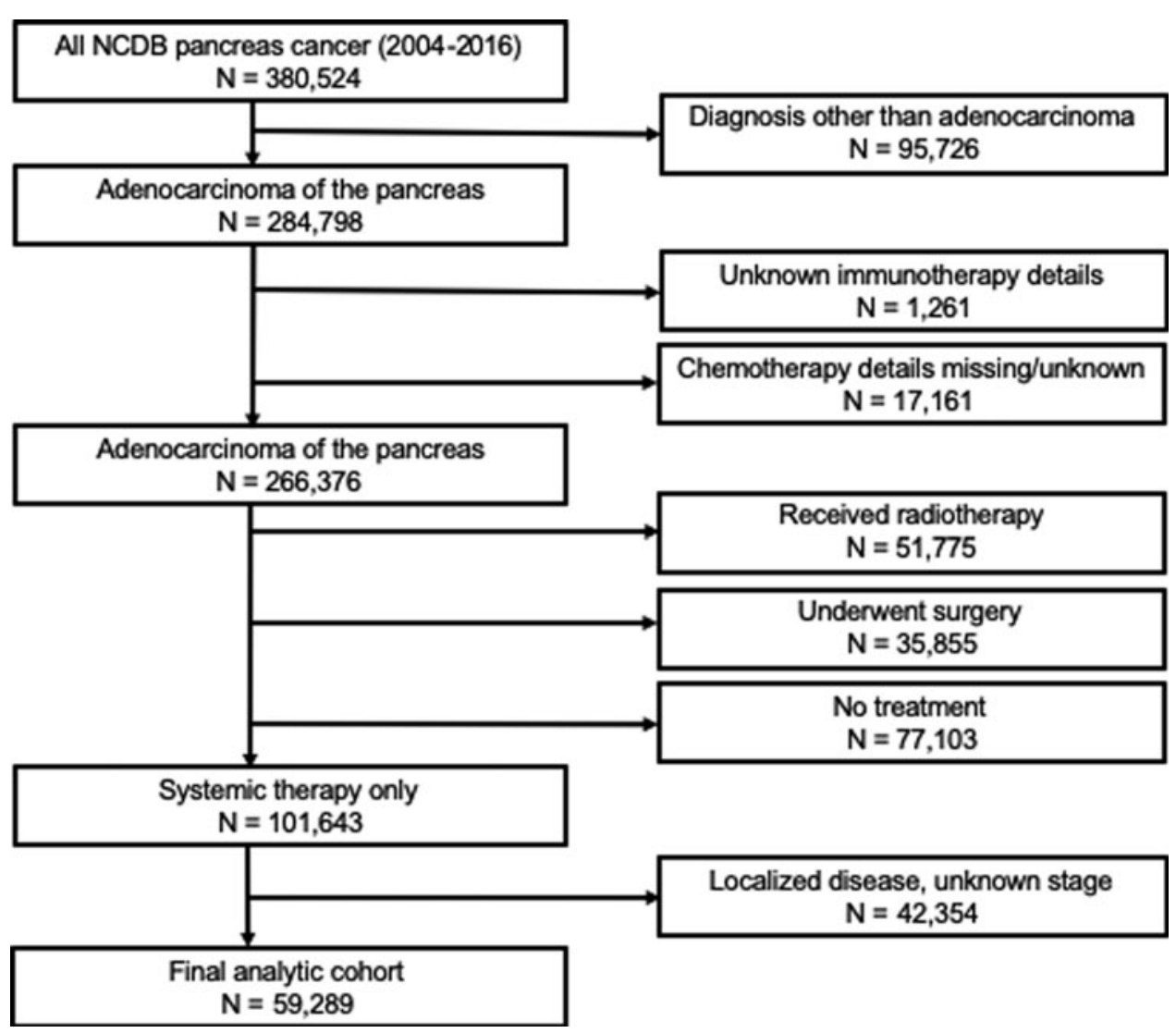

FIG. 1. Consort diagram of study population.

\section{Results}

Demographic and clinical data

A total of 59,289 patients were identified, of whom 58,947 (99.4\%) received CT and $342(0.6 \%)$ received IT-CT (Table 1). Patients who received IT-CT were younger (64 years vs. 66 years, $p=0.002$ ), more likely to be white, and more likely to have private insurance as compared with those who received CT. Patients who received IT-CT had fewer comorbidities as compared with patients who received CT (Charlson-Deyo score $=$ $0: 76.3 \%$ vs. $68.9 \%, p=0.007)$. The majority of patients in the IT-CT group were treated at academic hospitals (56.6\% IT-CT vs. $39.7 \%$ CT, $p<0.001$ ). Usage of multiagent CT was similar between the groups.

Factors associated with utilization of IT

Multivariable logistic regression was used to determine what factors were associated with the administration of IT (Table 2). Advanced age and greater comorbidity score were associated with a decreased likelihood of receiving IT. Treatment at an academic facility was associated with an increased likelihood of receiving IT (odds ratio $=1.95,95 \%$ CI $1.58-2.41$ ). There was no association between receipt of IT and sex, race, or insurance payor.

\section{Survival analyses}

Patients who received combined IT-CT had longer median overall survival than patients treated with CT alone (7.9 months vs. 6.3 months, $p=0.005$; Table 3 and Fig. 2). The 2-year survival rates were similar (IT-CT: $7.32 \%$, CT: $7.01 \%$ ). Of note, there was an additional group of patients who received IT without CT. This group $(n=17)$ had a median survival of 5.2 months.

Additionally, we performed a subgroup analysis for patients diagnosed in 2010 or later as both FOLFIRINOX and gemcitabine/nab-paclitaxel were introduced around that time. ${ }^{2,3} \mathrm{We}$ found that the median survival of patients who received IT-CT $(n=161)$ increased to 9.7 months and was longer than those who received CT only ( $n=32,666,6.8$ months, $p=0.017$, Fig. 3 ). 
Table 1. Summary of demographic and clinical data among patients with metastatic pancreatic ductal adenocarcinoma (National Cancer Database, 2004-2016)

\begin{tabular}{|c|c|c|c|}
\hline Characteristic & IT-CT $(n=342), n(\%)$ & CT $(n=58,947), n(\%)$ & $P$ \\
\hline Age, years, median (range) & $64(21-86)$ & $66(18-90)$ & 0.002 \\
\hline Male & $187(54.7)$ & $32,058(54.4)$ & 0.913 \\
\hline Race/Ethnicity & & & 0.008 \\
\hline White & $278(81.3)$ & $44,770(75.9)$ & \\
\hline Black & $21(6.1)$ & $6960(11.8)$ & \\
\hline Hispanic & $18(5.3)$ & $2465(4.2)$ & \\
\hline Other & $25(7.3)$ & $4752(8.1)$ & \\
\hline Primary insurance payor & & & 0.001 \\
\hline None & $4(1.2)$ & $1744(3.0)$ & \\
\hline Private & $168(49.1)$ & $23,236(39.4)$ & \\
\hline Medicare & $140(40.9)$ & $29,183(49.5)$ & \\
\hline Medicaid, other & $30(8.8)$ & $4784(8.1)$ & \\
\hline Education: $<6.3 \%$ of population w/o high school diploma & $113(33.5)$ & $15,862(27.3)$ & 0.031 \\
\hline Income: $\geq \$ 63,333$ annual household income & $151(45.1)$ & $22,149(38.2)$ & 0.001 \\
\hline Charlson-Deyo score $=0$ & $261(76.3)$ & $40,639(68.9)$ & 0.007 \\
\hline Facility type & & & $<0.001$ \\
\hline Community & $25(7.5)$ & $4744(8.1)$ & \\
\hline Comprehensive community & $97(29.0)$ & $22,899(39.3)$ & \\
\hline Academic/research & $189(56.6)$ & $23,126(39.7)$ & \\
\hline Integrated network & $23(6.9)$ & 7537 (12.9) & \\
\hline Distance to hospital, miles, median (IQR) & $17.8(6.7-46.6)$ & $10.1(4.5-24.2)$ & $<0.001$ \\
\hline Tumor size, mm, median (IQR) & $40(30-52)$ & $38(30-51)$ & 0.520 \\
\hline Multiagent $\mathrm{CT}$ & $228(66.7)$ & $37,043(62.8)$ & 0.144 \\
\hline Single-agent $C T$ & $114(33.3)$ & $21,904(37.2)$ & \\
\hline
\end{tabular}

Bold values indicate statistical significance.

$\mathrm{CT}$, chemotherapy; IQR, interquartile range; IT, immunotherapy.

A multivariable Cox regression analysis examining overall survival in the treatment groups is shown in Table 4. IT-CT was associated with a survival advantage relative to $\mathrm{CT}$ alone $(\mathrm{HR}=0.86,95 \%$ CI $0.76-0.97)$. Patients with advanced age, those treated at nonacademic facilities, and those with a greater CharlsonDeyo score also had an associated survival disadvantage. Those who received multiagent CT had an associated survival benefit as compared with those who received single-agent CT $(\mathrm{HR}=0.65,95 \% \mathrm{CI} 0.64-0.66)$.

Table 2. Multivariable logistic regression analyzing utilization of immunotherapy among patients with metastatic pancreatic ductal adenocarcinoma (National Cancer Database, 2004-2016)

\begin{tabular}{lccr}
\hline & OR & $95 \%$ Cl & \multicolumn{1}{c}{$\boldsymbol{p}$} \\
\hline Age & 0.99 & $0.98-1.00$ & $\mathbf{0 . 0 4 6}$ \\
Female vs. male & 1.01 & $0.82-1.25$ & 0.929 \\
White vs. other race & 1.30 & $0.99-1.71$ & 0.055 \\
Private insurance vs. other & 1.23 & $0.96-1.56$ & 0.100 \\
Top 2 income quartiles vs. bottom & 0.86 & $0.66-1.12$ & 0.277 \\
Top 2 education quartiles vs. bottom & 1.34 & $1.03-1.74$ & $\mathbf{0 . 0 3 1}$ \\
Academic facility vs. other & 1.95 & $1.58-2.41$ & $<\mathbf{0 . 0 0 1}$ \\
Charlson-Deyo score $\geq 1$ vs. 0 & 0.77 & $0.60-0.99$ & $\mathbf{0 . 0 4 0}$ \\
\hline
\end{tabular}

Bold values indicate statistical significance.

$95 \% \mathrm{Cl}, 95 \%$ confidence intervals; OR, odds ratio.

\section{Discussion}

In recent years, IT has revolutionized the field of oncology. Several malignancies in which IT has improved survival, such as melanoma, lung, and renal cell carcinoma, are well vascularized, highly immunogenic, have a high mutational burden, and have a microenvironment conducive to immune cell survival and function. ${ }^{29,30}$ Despite encouraging results in other malignancies, IT has not demonstrated efficacy in the majority of PDAC patients. More recently, olaparib (a PARP inhibitor) was approved for utilization in selected patients with metastatic pancreatic cancer. There was no difference in median survival as compared with placebo (18.9 months vs. 18.1 months), but olaparib was associated with a greater progression-free survival (7.4 months vs. 3.8 months). ${ }^{31}$ Of note, olaparib was only approved for patients with $B R C A$ (breast cancer

Table 3. Summary of median survival among patients with metastatic pancreatic ductal adenocarcinoma stratified by treatment group (National Cancer Database, 2004-2015)

\begin{tabular}{lcccc}
\hline Treatment & $\boldsymbol{n}$ & Median & IQR & $\boldsymbol{p}$ \\
\hline IT-CT & 268 & 7.9 & $4.8-13.1$ & 0.005 \\
CT & 52,344 & 6.3 & $3.2-11.7$ & \\
IT only & 17 & 5.2 & $2.7-15.5$ & -
\end{tabular}




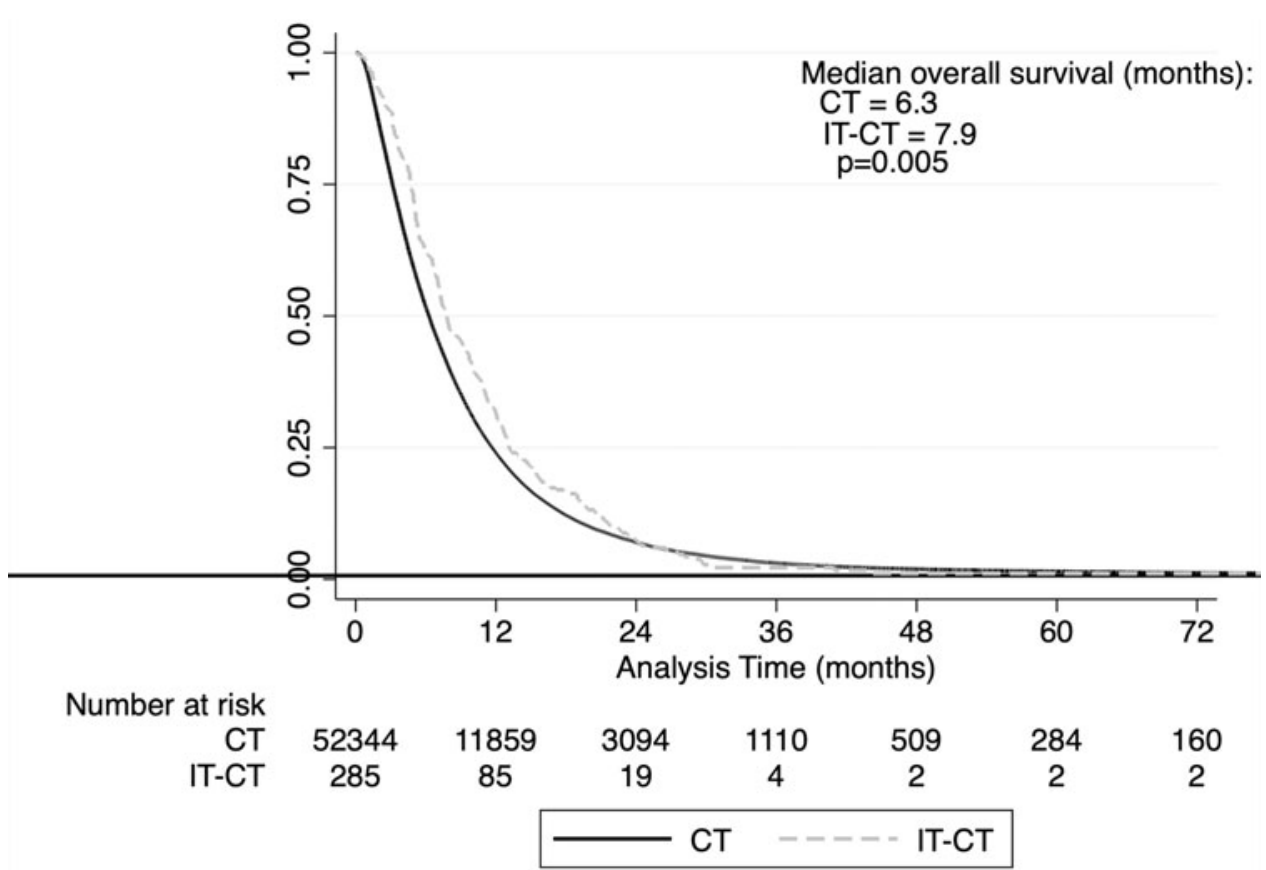

FIG. 2. Kaplan-Meier estimates of survival by treatment group (NCDB, 2004-2015). NCDB, National Cancer Database.
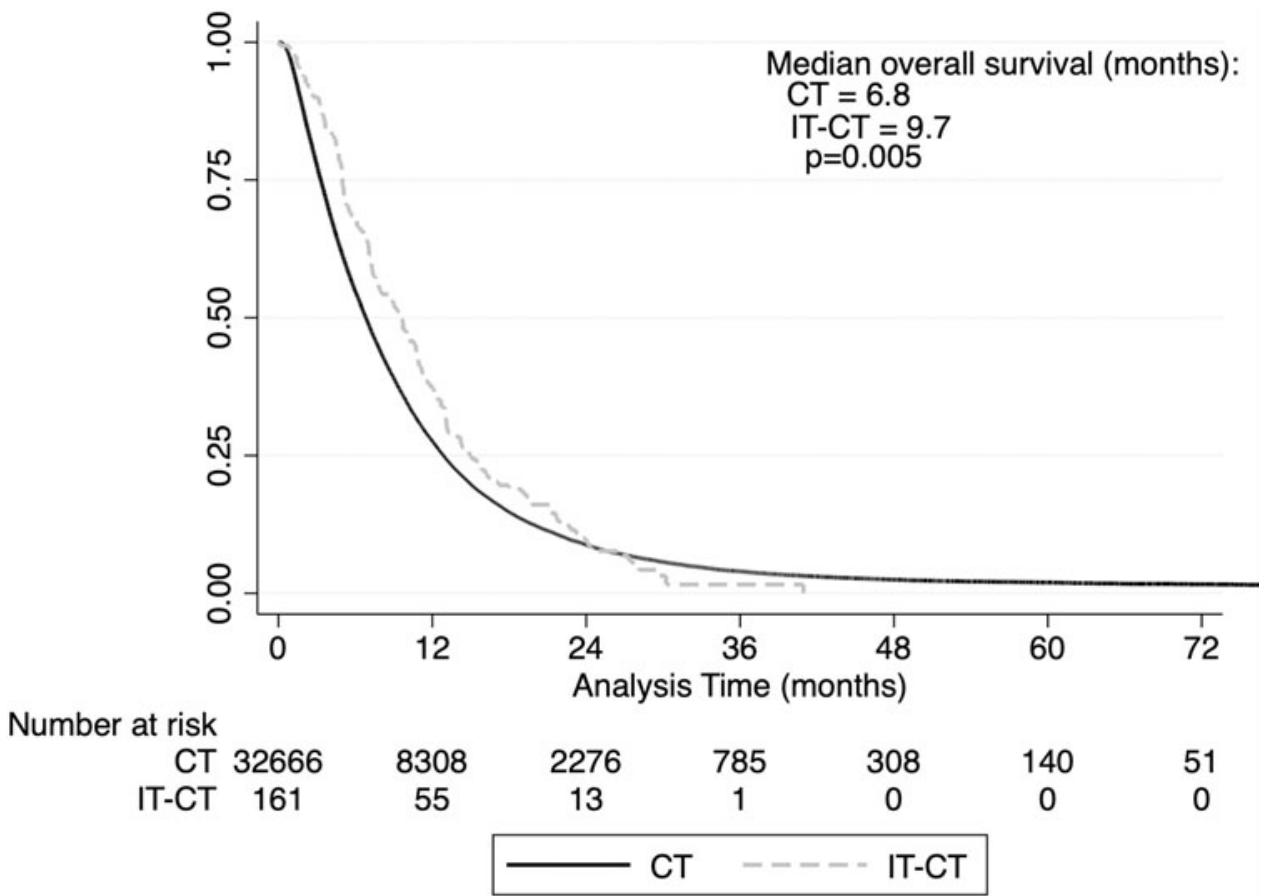

FIG. 3. Kaplan-Meier estimates of survival by treatment group (NCDB, 2010-2015). 
Table 4. Multivariable Cox proportional hazards regression analyzing overall survival between treatment groups (National Cancer Database, 2004-2015)

\begin{tabular}{lcrr}
\hline & HR & 95\% Cl & $\boldsymbol{p}$ \\
\hline IT-CT vs. CT & 0.86 & $0.76-0.97$ & 0.015 \\
Age & 1.01 & $1.00-1.01$ & $<0.001$ \\
Female vs. male & 0.93 & $0.91-0.95$ & $<0.001$ \\
Academic facility vs. other & 0.83 & $0.82-0.85$ & $<0.001$ \\
Private insurance vs. other & 0.94 & $0.92-0.96$ & $<0.001$ \\
Charlson-Deyo score $\geq 1$ vs. 0 & 1.13 & $1.11-1.15$ & $<0.001$ \\
Multiagent CT vs. single-agent CT & 0.65 & $0.64-0.66$ & $<0.001$ \\
\hline
\end{tabular}

$H R$, hazard ratio.

gene)-mutated pancreatic cancer, which accounts for just $5 \%$ of cases. ${ }^{32}$ The median survival of both arms in the aforementioned study was greater than the current analysis. To be included in this trial, patients had to complete 16 weeks of CT, likely preventing enrollment of patients with poor performance status or rapidly progressive disease. Therefore, results of the olaparib trial are likely not broadly generalizable to the majority of patients with metastatic PDAC.

In our study, we report that the addition of IT to CT was associated with a survival advantage in patients with metastatic PDAC. Median overall survival was $\sim 1.6$ months longer in the IT-CT group. This is concordant with a recent report which demonstrated that the combination of IT and CT in the adjuvant setting was associated with improved survival compared with adjuvant CT alone (5-year overall survival: $30.3 \%$ vs. $20.6 \%){ }^{27}$ The associated survival advantage among patients who received adjuvant IT-CT as compared with adjuvant $\mathrm{CT}$ was greater in magnitude relative to the findings in our study. We hypothesize that this discrepancy may be due to the effectiveness of treatments based on disease burden. For example, when compared with single-agent gemcitabine, FOLFIRINOX leads to a 19.4-month survival advantage in the adjuvant setting, but just a 4.3 -month advantage in metastatic PDAC.,5 It is possible that our current findings, as well as those reported in the adjuvant setting, are false-positive results, attributable to randomness in a relatively small sample size or unappreciated confounders.

Numerous immune targets have been studied in PDAC. A phase II trial in patients with metastatic PDAC who failed first-line CT received durvalumab, a monoclonal antibody targeting PD-L1 (programmed death-ligand 1); however, this trial was terminated due to a response rate of $0 \% .{ }^{23}$ Cytotoxic T lymphocyteassociated protein-4 (CTLA-4), another immune check- point, has been shown to downregulate immune responses. A single-arm clinical trial, which combined ipilimumab (a CTLA-4 antagonist), nivolumab (a PD-1 [programmed cell death protein 1] antagonist), and radiation in patients with metastatic PDAC demonstrated an objective response rate of just $14 \%$ and a progression-free survival of 76 days. ${ }^{24} \mathrm{~A}$ recent phase I trial examining the safety of nivolumab, nab-paclitaxel, and gemcitabine in patients with advanced PDAC did not show a survival benefit as compared with historic survival data without nivolumab. $^{33}$

Deficiencies in mismatch repair with subsequent microsatellite instability (MSI) are often found in cancers and cause numerous genetic mutations, activating a patient's antitumor immune response. ${ }^{34} \mathrm{~A}$ pivotal trial administered pembrolizumab (a PD-1 antagonist) to patients with metastatic cancer and demonstrated a disease control rate of $75 \%$ and a reported objective response rate of $62 \%$ in the limited subset of patients with pancreatic cancer $(n=8) .{ }^{35}$ A follow-up trial, which also administered pembrolizumab to MSI-high/mismatch repair-deficient advanced cancers, reported an objective response rate in tumors of pancreatic origin of just $18 \%$ with progression-free and overall survival of 2 and 4 months, respectively. ${ }^{22}$ Importantly, only $1 \%$ to $2 \%$ of PDAC tumors are MSI-high and/or mismatch repair deficient. ${ }^{36}$ It should be emphasized that the impact of IT on PDAC with MSI remains unproven, and the incidence of these tumors is extremely rare. One potential explanation for the discrepancy in effectiveness of IT relative to other cancers is due to the austere tumor microenvironment of PDAC, which includes a dense stromal compartment, low nutrient concentrations, and relative hypoxia. ${ }^{20,37}$ Patients' immune cells, which immunotherapies rely upon, are likely less effective under these harsh conditions, since they are not well adapted to survive in this harsh environment. ${ }^{37,38} \mathrm{~A}$ recent study by our group demonstrated that among all active phase III trials in the United States, 37\% targeted the immune system. ${ }^{39}$ While our data show that some patients benefit from IT, the 2-year survival rate was just $7 \%$. Thus, ongoing research efforts may want to investigate other potential targets.

Our study also highlights disparities in utilization of IT. We found that advanced age and greater comorbidity index were associated with a decreased utilization of IT. Higher level education and receiving treatment at an academic center were also associated with increased IT utilization. Disparities in cancer are well studied and previous reports have shown variations in therapies 
administered and survival based on race and socioeconomic status. ${ }^{40,41}$ Studies have recommended multiple strategies, including insurance reform, improved access to quality care with community outreach, improved access to novel therapies, increased emphasis on prevention and screening, and diversification of employees in the health care field, to reduce disparities in cancer care. ${ }^{42-44}$ Previous reports identified that Black and Asian patients, those who live alone, were unmarried or did not have children, had government-issued insurance, or had issues performing their activities of daily living were more likely to decline all treatment. ${ }^{45,46}$

Although our study includes data on a large number of PDAC patients, there are limitations. Importantly, the use of IT is not common, therefore the IT-CT treatment group is quite small in comparison to those who received $\mathrm{CT}$ alone. Those who received IT likely represent a highly select patient cohort, which may not be generalizable to all patients with metastatic PDAC. We attempted to control for this by including appropriate variables (age, insurance payor, treating facility type, and Charlson-Deyo comorbidity index) in our multivariable models. Based on limitations inherent to NCDB studies, including the lack of granularity of the data, we cannot comment on specific treatment details, such as the type of CT or IT (oncolytic virus, cancer vaccine, immune checkpoint inhibitors) received, whether the IT was part of a clinical trial, how many cycles of CT were completed, the genetic make-up of a patient's tumor (BRCA mutation, MSI status, etc.), and why IT was administered (progression of disease, patient desire, clinical trial, etc.). Also, the NCDB only includes data on the first 6 months of treatment, therefore a subset of patients in the CT group may have received IT later in their treatment course. Details of a patient's performance status are not recorded in the NCDB, thus Charlson-Deyo scores were used as a surrogate. The NCDB only receives data from the Commission on Cancer-accredited hospitals and only captures $70 \%$ of cancer diagnoses in the United States. Therefore, these data cannot truly be generalized to all hospital systems and patients. Despite these limitations, our study reports a thorough analysis of the utilization of IT in patients with metastatic PDAC and demonstrates there may be a subset of patients who respond to IT.

\section{Conclusion}

In patients with metastatic PDAC, the addition of IT to CT was associated with improved median survival but a similar 2-year survival rate. Future research is needed to identify strategies that both stimulate entry of antitumor immune cells into the PDAC microenvironment and increase the antitumor capabilities of these cells.

\section{Authors' Contributions}

J.J.H.- conceptualization, data curation, formal analysis, writing - original draft. K.B.-writing-review and editing. K.S. - writing-review and editing. S.C.M.-formal analysis, writing - review and editing. L.D.R.-writingreview and editing. J.M.H.-writing-review and editing. J.B.A.—writing-review and editing. J.M.W.conceptualization, supervision, writing-review and editing. L.M.O.-supervision, writing-original draft, writing-review and editing.

\section{Preprint}

An earlier draft of this article was posted at Research Square (DOI: 10.21203/rs.3.rs-121110/v1).

\section{Author Disclosure Statement}

No competing financial interests exist.

\section{Funding Information}

No funding was received for this article.

\section{References}

1. NCCN guidelines version 1. 2019 pancreatic adenocarcinoma. National Comprehensive Cancer Network. Available at https://www.nccn.org/ professionals/physician_gls/PDF/pancreatic.pdf Accessed March 9, 2020

2. Conroy $T$, Desseigne $F$, Ychou $M$, et al. FOLFIRINOX versus gemcitabine for metastatic pancreatic cancer. N Engl J Med. 2011;364:1817-1825.

3. Von Hoff DD, Ervin T, Arena FP, et al. Increased survival in pancreatic cancer with nab-paclitaxel plus gemcitabine. N Engl J Med. 2013;369: 1691-1703.

4. Cunningham D, Chau I, Stocken DD, et al. Phase III randomized comparison of gemcitabine versus gemcitabine plus capecitabine in patients with advanced pancreatic cancer. J Clin Oncol. 2009;27:5513-5518.

5. Conroy T, Hammel P, Hebbar M, et al. FOLFIRINOX or gemcitabine as adjuvant therapy for pancreatic cancer. N Engl J Med. 2018;379:2395-2406.

6. Powles T, Eder JP, Fine GD, et al. MPDL3280A (anti-PD-L1) treatment leads to clinical activity in metastatic bladder cancer. Nature. 2014;515:558564.

7. Choueiri TK, Xie W, Kollmannsberger C, et al. The impact of cytoreductive nephrectomy on survival of patients with metastatic renal cell carcinoma receiving vascular endothelial growth factor targeted therapy. J Urol. 2011;185:60-66.

8. Dobry AS, Zogg CK, Hodi FS, et al. Management of metastatic melanoma: improved survival in a national cohort following the approvals of checkpoint blockade immunotherapies and targeted therapies. Cancer Immunol Immunother. 2018;67:1833-1844.

9. Rizvi NA, Mazières J, Planchard $D$, et al. Activity and safety of nivolumab, an anti-PD-1 immune checkpoint inhibitor, for patients with advanced, refractory squamous non-small-cell lung cancer (CheckMate 063): a phase 2, single-arm trial. Lancet Oncol. 2015;16:257-265.

10. Kunk PR, Bauer TW, Slingluff CL, et al. From bench to bedside a comprehensive review of pancreatic cancer immunotherapy. J Immunother Cancer. 2016;41-12.

11. Immunotherapy to treat cancer. National Cancer Institute. Available at https://www.cancer.gov/about-cancer/treatment/types/immunotherapy Accessed March 9, 2020. 
12. Manji GA, Olive KP, Saenger YM, et al. Current and emerging therapies in metastatic pancreatic cancer. Clin Cancer Res. 2017;23:1670-1678.

13. Torphy RJ, Zhu Y, Schulick RD. Immunotherapy for pancreatic cancer: barriers and breakthroughs. Ann Gastroenterol Surg. 2018;2:274-281.

14. Guo $S$, Contratto M, Miller G, et al. Immunotherapy in pancreatic cancer: unleash its potential through novel combinations. World J Clin Oncol. 2017;8:230-240.

15. Jaffee EM, Hruban RH, Biedrzycki B, et al. Novel allogeneic granulocytemacrophage colony-stimulating factor-secreting tumor vaccine for pancreatic cancer: a phase I trial of safety and immune activation. J Clin Oncol. 2001;19:145-156.

16. Laheru D, Lutz E, Burke J, et al. Allogeneic granulocyte macrophage colony-stimulating factor-secreting tumor immunotherapy alone or in sequence with cyclophosphamide for metastatic pancreatic cancer: a pilot study of safety, feasibility, and immune activation. Clin Cancer Res. 2008;14:1455-1463.

17. Gjertsen MK, Buanes $T$, Rosseland AR, et al. Intradermal ras peptide vaccination with granulocyte-macrophage colony-stimulating factor as adjuvant: clinical and immunological responses in patients with pancreatic adenocarcinoma. Int J Cancer. 2001;92:441-450.

18. Hecht JR, Bedford R, Abbruzzese JL, et al. A phase I/II trial of intratumora endoscopic ultrasound injection of ONYX-015 with intravenous gemcitabine in unresectable pancreatic carcinoma. Clin Cancer Res. 2003;9: $555-561$.

19. Hodi FS, O'Day SJ, McDermott DF, et al. Improved survival with ipilimumab in patients with metastatic melanoma. N Engl J Med. 2010;363:711723.

20. Looi CK, Chung FFL, Leong CO, et al. Therapeutic challenges and current immunomodulatory strategies in targeting the immunosuppressive pancreatic tumor microenvironment. J Exp Clin Cancer Res. 2019;38:1-23.

21. Hilmi M, Bartholin L, Neuzillet C. Immune therapies in pancreatic ductal adenocarcinoma: where are we now? World J Gastroenterol. 2018;24: 2137-2151.

22. Marabelle A, Le DT, Ascierto PA, et al. Efficacy of pembrolizumab in patients with noncolorectal high microsatellite instability/mismatch repairdeficient cancer: results from the phase II KEYNOTE-158 study. J Clin Oncol. 2019;38:1-10.

23. O'Reilly EM, Oh D-Y, Dhani N, et al. Durvalumab with or without tremelimumab for patients with metastatic pancreatic ductal adenocarcinoma: a phase 2 randomized clinical trial. JAMA Oncol. 2019;5:1431-1438.

24. Parikh A, Wo JY-L, Ryan DP, et al. A phase II study of ipilimumab and nivolumab with radiation in metastatic pancreatic adenocarcinoma. J Clin Oncol. 2019;37(4 Suppl):391.

25. Brahmer JR, Tykodi SS, Chow LQM, et al. Safety and activity of anti-PD-L1 antibody in patients with advanced cancer. N Engl J Med. 2012;366:24552465.

26. Wainberg ZA, Hochster HS, Kim EJ, et al. Open-label, phase I study of nivolumab combined with nab-paclitaxel plus gemcitabine in advanced pancreatic cancer. Clin Cancer Res. 2020;26:4814 LP-4822 LP.

27. Tran TB, Maker VK, Maker AV. Impact of immunotherapy after resection of pancreatic cancer. J Am Coll Surg. 2019;229:19-27.e1

28. The National Cancer Database 2016 PUF Data Dictionary. American College of Surgeons. Available at https://www.facs.org/-/media/files/qualityprograms/cancer/ncdb/puf_data_dictionary_2016.ashx Accessed March 11, 2021.

29. Lugowska I, Teterycz P, Rutkowski P. Immunotherapy of melanoma. Contemp Oncol. 2018;22:61-67.

30. Santoni M, Massari F, Di Nunno V, et al. Immunotherapy in renal cell carcinoma: latest evidence and clinical implications. Drugs Context. 2018; 7:212528.

31. Golan $T$, Hammel $P$, Reni $M$, et al. Maintenance olaparib for germline BRCAmutated metastatic pancreatic cancer. N Engl J Med. 2019;381:317-327.

32. Holter S, Borgida A, Dodd A, et al. Germline BRCA mutations in a large clinic-based cohort of patients with pancreatic adenocarcinoma. J Clin Oncol. 2015;33:3124-3129.
33. Wainberg ZA, Hochster HS, Kim EJ, et al. Open-label, phase i study of nivolumab combined with nab-paclitaxel plus gemcitabine in advanced pancreatic cancer. Clin Cancer Res. 2020;26:4814-4822.

34. Zhao $P$, Li L, Jiang $X$, et al. Mismatch repair deficiency/microsatellite instability-high as a predictor for anti-PD-1/PD-L1 immunotherapy efficacy. J Hematol Oncol. 2019;12:54.

35. Le DT, Durham JN, Smith KN, et al. Mismatch repair deficiency predicts response of solid tumors to PD-1 blockade. Science. 2017;357:409-413.

36. Ahmad-Nielsen SA, Bruun Nielsen MF, Mortensen MB, et al. Frequency of mismatch repair deficiency in pancreatic ductal adenocarcinoma. Pathol Res Pract. 2020;216:152985.

37. Vaziri-Gohar A, Zarei M, Brody JR, et al. Metabolic dependencies in pancreatic cancer. Front Oncol. 2018;8:617.

38. Bonaventura $\mathrm{P}$, Shekarian T, Alcazer V, et al. Cold tumors: a therapeutic challenge for immunotherapy. Front Immunol. 2019;10:168.

39. Katayama E, Hue JJ, Bajor DL, et al. A comprehensive analysis of clinical trials in pancreatic cancer: what is coming down the pike? Oncotarget. 2020;11:3489-3501.

40. O'Keefe EB, Meltzer JP, Bethea TN. Health disparities and cancer: racial disparities in cancer mortality in the United States, 2000-2010. Front Public Health. 2015;3:51.

41. Ward $E$, Jemal A, Cokkinides V, et al. Cancer disparities by race/ethnicity and socioeconomic status. CA Cancer J Clin. 2004;54:78-93.

42. Kagawa-Singer M, Valdez Dadia A, Yu MC, et al. Cancer, culture, and health disparities: time to chart a new course? CA Cancer J Clin. 2010;60:12-39.

43. Chin $M H$, Walters $A E$, Cook SC, et al. Interventions to reduce racial and ethnic disparities in health care. Med Care Res Rev. 2007;64(5 Suppl):7-28.

44. Moy B, Polite BN, Halpern MT, et al. American Society of Clinical Oncology policy statement: opportunities in the patient protection and affordable care act to reduce cancer care disparities. J Clin Oncol. 2011;29:38163824.

45. Puts MTE, Monette J, Girre V, et al. Characteristics of older newly diagnosed cancer patients refusing cancer treatments. Support Care Cancer. 2010;18:969-974

46. Restrepo DJ, Sisti A, Boczar D, et al. Characteristics of breast cancer patients who refuse surgery. Anticancer Res. 2019;39:4941-4945.

Cite this article as: Hue J, Bingmer K, Sugumar K, Markt SC, Rothermel LD, Hardacre JM, Ammori JB, Winter JM, Ocuin LM (2021) Immunotherapy is associated with a survival benefit in patients receiving chemotherapy for metastatic pancreas cancer, Journal of Pancreatic Cancer 7:1, 31-38, DOI: 10.1089/pancan.2021.0003. 